\title{
Einwirkung von Ammoniak auf Methylaethylacroleïn
}

\author{
von
}

Fduard Hoppe.

\begin{abstract}
Aus dem chemischen Laboratorium des Prof. Ad. Lieben an der k. k. Universität in Wien.
\end{abstract}

(Vorgelegt in der Sitzung am 5. Juli 1888.)

Von den Homologen des Acetaldehyds gibt ein Theil bei der Einwirkung von Ammoniak directe Additionsproducte, so der Acetaldehyd selbst, der Butyraldehyd, der Isovaleraldehyd, der Oenanthaldehyd und der Propionaldehyd; ein anderer Theil dieser gesättigten Aldehyde liefert Alkyliden-Polyamine, complicirt zusammengesetzte Verbindungen, welche sich von mehreren Molekülen Ammoniak ableiten, in welchen der Wasserstoff äquivalent durch Alkyliden ersetzt ist.

Zu diesen gehört der Formaldehyd, aus welchem das Hexamethylentetramin ${ }^{1}\left(\mathrm{CH}_{2}\right)_{6} \mathrm{~N}_{4}$ entsteht und der Isobutyraldehyd, aus welchem das Oxyheptaisobutylidenamin ${ }^{2} \mathrm{~N}_{6}\left(\mathrm{C}_{4} \mathrm{H}_{8}\right)_{7} \mathrm{H}_{6} \mathrm{O}$ entsteht, das beim Erhitzen in Ammoniak, Isobutyraldehyd und Triisobutylidendiamin ${ }^{3}\left(\mathrm{C}_{4} \mathrm{H}_{8}\right)_{3} \mathrm{~N}_{2}$ oder - wenn die Erhitzung langsam vorgenommen wird - in Isobutenylbutylidenamin ${ }^{3}$ $\mathrm{C}_{8} \mathrm{H}_{15} \mathrm{~N}$ zerfällt.

Auch der Oenanthaldehyd liefert ein Trioenanthylidendiamin $^{4}\left(\mathrm{C}_{17} \mathrm{H}_{14}\right)_{3} \mathrm{~N}_{2}$ und der Acetaldehyd das Hydracetamid ${ }^{5}$ $\mathrm{C}_{6} \mathrm{H}_{12} \mathrm{~N}_{2}$.

1 Butlerow: Ann. 115, 322.

2 Lipp: Ann. $205,4$.

3 Lipp: Ann. 211, 345 und 349.

4 Schiff: Ann. Spl. 3, 367.

5 Schiff: Ann. Spl. 6, 1; Strecker: Ann. Spl. 6, 255. 
Die ảrecten Additionsproducte sind sämmtlich mehr oder weniger unbeständig, schon durch den Einfluss verdünnter Säuren in der Wärme zersetzlich und geben bemerkenswertherweise, wenn man sie erhöhten Temperaturen aussetzt, unter Ammoniakabspaltung Körper, welche als Homologe des Pyridin anzusprechen sind.

So haben Ador und Baeyer ${ }^{1}$ ans Aldehydammoniak Aldehydcollidin und daneben Paracollidin erhalten und Vohl ${ }^{2}$ diese Angaben bestätigt; desgleichen erhielten $D$ ürkopf and Schlaugk ${ }^{3}$ durch Erhitzen von Aldehydammoniak und Paraldehyd ein Collidin.

Aus Propionaldehydammoniak erhält man nach $W$ aage ${ }^{4}$ beim Erhitzen ein Parvolin $\mathrm{C}_{9} \mathrm{H}_{13} \mathrm{~N}$, welches tibrigens auch - wie Plöehl ${ }^{5}$ ankündigt - entstehen soll, wenn man Propionaldehyd in eine Salmiaklösung einträgt; ein Parvolin wurde auch von Dürkopf und Schlaugk ${ }^{\dagger}$ dargestellt durch Erhitzen von je einem Molekïl Propionaldehydammoniak mit je drei Molekülen Paraldebyd.

Butyraldehyd liefert, wie Schiff ${ }^{7}$ behauptet, mit alkoholischem Ammoniak auf $100^{\circ}$ erhitzt, zunächst eine Base, welche our in ihrem Platindoppelsalze $\left(\mathrm{C}_{16} \mathrm{H}_{29} \mathrm{NO} . \mathrm{HCl}\right)_{2} \mathrm{PtCl}_{4}$ fassbar ist, und freigemacht sofort in Wasser und Paraconiin zerfällt.

Aus Isovaleraldehyd entsteht bei Einwirkung von Ammoniak bei hoher Temperatur Valeritrin, welchem Liubawin ${ }^{8}$ die Formel $\mathrm{C}_{15} \mathrm{H}_{27} \mathrm{~N}$ zuschreibt.

Die Homologen des Acrylaldehyds sind viel weniger eingehend studirt und nur soviel scheint festzustehen, dass sie die einfachen Ammoniakadditionsproducte, wie wir sie beim Acetaldehyd, Butyraldehyd u. s. w. beobachten können, nicht liefern.

1 Ann. 155, 297 u. 306.

2 Jahresber. 1870, 807.

3 Berl. Ber. 18,920 u. 3432.

4 Monatsh. f. Chemie IV, 708.

5 Berl. Ber. 20, 722.

- Berl. Ber. 21, 386.

7 Ann. 157, 352.

8 Berl. Ber. 5, 1101 u. 6. 1460. 
Denn schon die einfachst zusammengesetzte Verbindung, welche aus einem ungesättigten Aldehyd und Ammoniak erhalten werden konnte, das Acrolë̈nammoniak, ist kein einfaches Additionsproduct mehr, sondern offenbar aus einem solchen durch Condensation entstanden. Doch erhält man hieraus analog den einfachen Aldehydammoniakverbindungen beim Erhitzen unter Wasserabspaltung ein Homologes des Pyridin: das Picolin (Baeyer ${ }^{1}$ ). Claus ${ }^{2}$ fand, dass das Acroleïnammoniak beim Erhitzen unter Ammoniakabspaltung in eine Base $\mathrm{C}_{12} \mathrm{H}_{19} \mathrm{NO}_{2}$ übergeführt werden könne; auch aus dem Crotonaldehyd, allerdings bei einer Abkühlung auf $-20^{\circ}$, erhielt Combes ${ }^{3}$ eine sauerstoff hältige Base $\mathrm{C}_{8} \mathrm{H}_{16} \mathrm{~N}_{2} \mathrm{O}$.

Anderseits aber entsteht aus dem Crotonaldehyd - analog den Alkyliden-Polyaminen der gesättigten Aldehyde - das von Würtz $\mathrm{z}^{4}$ dargestellte Tricrotonylenamin: $\mathrm{C}_{12} \mathrm{H}_{24} \mathrm{~N}_{4}+6 \mathrm{H}_{2} \mathrm{O}$.

Einer Aufforderung des Herrn Prof. Lieb en folgend, versuchte ich, anknüpfend an frühere Versuche W a ag e ${ }^{5}$, Ammoniak auf Methylaethylacroleïn einwirken zu lassen. Die Versuche beim Einleiten von trockenem Ammoniakgas in den Aldehyd oder in eine ätherische Lösung desselben bei $0^{\circ}$ ein Additionsproduct zu erhalten, hatten nicht den gewünschten Erfolg. Beim Erhitzen auf $100^{\circ} \mathrm{C}$. mit alkoholischem Ammoniak erhielt ich ein Product, welches jedoch so leicht zersetzlich ist, dass es mir trotz vieler Mühe nicht gelang, eines bestimmten einheitlichen Körpers habhaft zu werden.

Beim Erhitzen auf $200^{\circ} \mathrm{C}$. entstand Parvolin und daneben eine Base $\mathrm{C}_{12} \mathrm{H}_{19} \mathrm{~N}$, die vielleicht auch ein Homologes des Pyridin sein könnte; auch gelang es mir, das Platinsalz eines Picolin zu erhalten und zu bestimmen.

Die Constitution dieses Parvolin, das mit dem Wage's identisch ist, vermochte ich insoweit zu ermitteln, dass die Stellung der Seitenketten im Pyridinring klargestellt wird. Bei der Oxydation lieferte dieses Parvolin nämlich eine Dicarbonsäure, welche die rothe Eisenreaction $(\alpha)$ und beim Zerfall

1 Ann. 155, 283.

2 Ann. 158, 222.

3 Jahresber. 1883, 650 .

4 Bul. d. 1. soc. ehem. 34, 486 .

5 Monatsh. f. Chemie IV, 725. 
Nicotinsäure gibt. Die angenfällige Verschiedenheit dieser Dicarbonsäure von der Chinolinsäure zwingt zur Annahme, dass Isocinchomeronsäure vorliege, so dass das Parvolin die Seitenketten in der $\alpha$ und $\beta^{\prime}$ Stellung führen muss.

\section{Darstellung des Aldehyds.}

Ans Propylalkohol stellte ich in der von Lieben und Ze ise $1^{1}$ angegebenen Weise Propionaldehyd dar, die Ausbeute betrug, bei jedesmaliger Verarbeitnng von $200 \mathrm{~g}$ Propylalkohol, $164 g$ Kaliumbichromat und $218 g$ Schwefelsäure, 70 Procent des angewandten Propylalkohols.

Die Condensation des Propionaldehyd zu Methylaethylacrolein nahm ich, dem Beispiele von J. G. Schmidt ${ }^{2}$ und G. A. Raupenstrauch ${ }^{3}$ folgend, mit Natronlauge als Wasser entziehendem Agens vor. Das Verfahren, welches ich nach mehreren Versuchen als das ergiebigste beibehielt, war folgendes:

Je $50 \mathrm{~g}$ Propionaldehyd wurden mit $1000 \mathrm{~g}$ Wasser in einer Stöpselflasche zusammengebracht und mit $50 \mathrm{~cm}^{3}$ einer zehnprocentigen Natronlauge versetzt, geschüttelt und dann 40 Stunden stehen gelassen.

Anfangs bildet sich eine Emulsion, die unter gleichzeitiger Gelbfärbung der Flüssigkeit langsam schwindet, bis sich endlich zwei klare Schichten gebildet haben. Sodann wurde verdiunnte Schwefelsäure zugesetzt bis zur eben noch neutralen Reaction und mit Wasserdampf destillirt. Das im Destillate obenaufschwimmende Öl abgehoben, und in Kohlensäure-Atmosphäre mehrfach fractionirt, ergab $263 \mathrm{~g}$ reines zwischen $135-140^{\circ} \mathrm{C}$. übergegangenes Methylaethylacroleïn, erhalten ans $350 \mathrm{~g}$ Rohmaterial, respective aus $590 \mathrm{~g}$ Aldehyd. Diese Ausbeute beträgt $44 \cdot 6 \%$ gegen $84 \cdot 5 \%$ der theoretischen $\left(2 \mathrm{C}_{3} \mathrm{H}_{6} \mathrm{O}=\right.$ $=\mathrm{C}_{6} \mathrm{H}_{10} \mathrm{O}+\mathrm{H}_{2} \mathrm{O}$ ), bleibt also hinter der von Lieben und $\mathrm{Z}$ e ise $1^{4}$ bei Anwendung von Natriumacetat erzielten beträchtlich zuriick, die Darstellung aber ist bedeutend einfacher und rascher.

1 Monatsh. f. Chemie IV, 14.

2 Berl. Ber. 13, 2342 u. 14, 1459.

3 Monatsh. f. Chemie VIII, 112.

4 Monatsh. f. Chemie IV, 16. 
In die trockene ätherischeLösung des so gewonnenen Methylaethylacroleïns wurde bei $0^{\circ} \mathrm{C}$. Ammoniak eingeleitet; es entstand ein weisser, flockiger Körper; der sich jedoch im Eise schon zu zersetzen schien, indem die Menge der ausgeschiedenen Verbindung zusehends abnahm. Bei gewöhnlicher Temperatur verschwanden die Flocken ganz unter Abscheidung von Wasser. Ich verzichtete daher auf die Isolirung dieses höchst zersetzlichen Körpers.

\section{Einwirkung von Ammoniak bei $100^{\circ} \mathrm{C}$.}

Methylaethylacroleïn schloss ich in Partien von je $5 g$ mit einem Überschusse einer in der Kälte gesättigten alkoholischen Ammoniaklösung (zehnprocentig bei $0^{\circ}$ ) in Rohre ein, welche sechs Stunden lang auf $100^{\circ}$ erhitzt wurden.

Nachdem ich den Alkohol aus dem Wasserbade von dem Röhreninhalt abdestillirt hatte, stellte sich die Ausbente aus den $263 g$ Methylaethylacroleïn auf $300 g$ Rohproduct. Dieses Rohproduct vom Alkohol und Wasser durch Erhitzen bis zu $50^{\circ} \mathrm{im}$ Vacuum befreit und getrocknet, stellt sich als eine tiberaus dicke, zähe, gelbe, alkalische Flüssigkeit dar, welche stark bitter schmeckt und einen Geruch anfweist, der an den des Parvolin erinnert.

Die Analysen ergaben:

I. $0 \cdot 3859 \mathrm{~g}$ Substanz lieferten bei der Verbrennung $0.9717 g$ Kohlensäre und $0.3952 g$ Wasser.

II. $0 \cdot 2215 \mathrm{~g}$ Substanz lieferten bei der Verbrennung $0.5545 \mathrm{~g}$ Kohlensäure und $0 \cdot 2330 \mathrm{~g}$ Wasser.

III. $0 \cdot 1794 g$ Substanz lieferten bei der Stickstoff bestimmung nach D umas $0.0330 \mathrm{~g}$ Stickstoff.

VI. $0 \cdot 2569 g$ Substanz lieferten bei der Stiekstoffbestimmung nach Dumas $0.0468 g$ Stickstoff.

Bei der Destillation im Vacuum von $17 \mathrm{~mm}$ Druck ergab es sich, dass der weitaus grösste Theil der Substanz zwischen 205 und $209^{\circ} \mathrm{C}$. glatt überging, jedoch war deutlich Wasserausscheidung und Ammoniakreaction in einem zwischen der Pumpe und dem Destillationsapparate angebrachten Zwischengefässe bemerkbar. 
Dieses offenbar unter geringer Zersetzung entstandene Product ergab vacuumtrocken die folgenden Analysenzahlen:

V. $0.3573 g$ Substanz lieferten bei der Verbrennung $0 \cdot 8780 \mathrm{~g}$ Kohlensäure und $0 \cdot 3573 \mathrm{~g}$ Wasser.

VI. $0 \cdot 2858 g$ Substanz lieferten bei der Verbrennung: $0 \cdot 7371 \mathrm{~g}$ Kohlensäure und $0 \cdot 2925 \mathrm{~g}$ Wasser.

VII. $0 \cdot 2309 \mathrm{~g}$ Substanz lieferten bei der Verbrennung $0.5983 g$ Kohlensäure und $0.2309 g$ Wasser.

VIII. $0 \cdot 2455 g$ Substanz lieferten bei der Stickstoff bestimmung nach Dumas $0.0396 g$ Stickstoff.

IX. $0 \cdot 3017 g$ Substanz lieferten bei der Stickstoffbestimmung nach D u mas $0.0488 g$ Stickstoff.

In 100 Theilen:

Gefunden:

undestillirtes Product, destillirtes Product

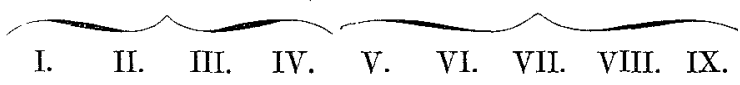

Kohlenstoff: ....68.67 68.27 — -69.9770 .3370 .67 -

Wasserstoff:.... 11.3811 .69 - $\quad$ - $11.6011 .3711 .70 \quad$ -

Stickstoff: ..... - $\quad$ - 18.3918 .26 - $\quad$ - 16.1816 .11

Es ist hieraus deutlich zu sehen, dass durch die Destillation und durch die damit verbundene Erwärmung auf $200^{\circ}$, wenn sie auch nur kurze Zeit währte - eine Veränderung der Substanz vor sich geht, in dem Sinne, dass ihr Kohlenstoffgehalt zu-, ihr Stickstoffgehalt abnimmt. Indess stehen die Zahlen des undestillirten und destillirten Productes doch zu nahe, als dass man an eine vollständige oder auch nur sehr weitgehende Zersetzung des Rohproductes zu denken veranlasst wäre.

Ausserdem aber zeigen die Analysen des undestillirten und des destillirten Productes einen Abgang von $1 \frac{1}{2}$ bis $2 \%$, welcher wohl nicht nur auf Analysenfehler zurückzuführen ist, sondern anf die Beimengung eines sauerstoffhältigen Körpers hinzudenten schien.

Dies veranlasste mich, das Rohproduct in Salzsäure zu lösen. Die salzsauere Lösung ist rothbraun, und der Geruch nach dem Condensationsproducte $\mathrm{C}_{6} \mathrm{H}_{10} \mathrm{O}$ allerdings nur in geringem 
Masse bemerkbar, ein Zeichen, dass die Substanz entweder eine Veränderung erfährt, oder - was allerdings unwahrscheinlich ist - noch Methylaethylacroleïn enthalten hatte. Die salzsanere Lösung schüttelte ich mit $\ddot{A}$ ther aus, schied und behielt nach Abdestilliren des Äthers eine rothbraune Flüssigkeit von angenehmem Geruche. Das aus der chlorwasserstoffsaueren Lösung mittels Ätzkali frei gemachte basische Product wurde, um eine etwaige Zersetzung beim Erwärmen zu vermeiden, mit Äther aufgenommen und nach Abdunstung desselben im Vactum getrocknet und den folgenden Reactionen und Analysen unterworfen:

Die schwachsalzsauere Lösung des Productes gibt mit:

Platinchlorid: nach längerer Zeit harzigen Niederschlag;

Goldchlorid: öligen, gelben Niederschlag;

Quecksilberchlorid: weisse Trübung;

Pikrinsäure: amorphen, gelben Niederschlag;

Jodjodkalium: amorphen, braunen Niederschlag;

Kaliumquecksilberjodid: amorphen, gelblichweissen, starken Niederschlag;

Cadmiumjodid: amorphen, weissen Niederschlag;

Phosphorwolframsäure: amorphen, weissen, massigen Niederschl.; Phosphormolybdänsäure: amorphen, strohgelben, dicken Niederschlag;

Kaliumnitrit: schmutzig weissen Niederschlag, welcher in verdünnter Säure unlöslich ist; Äther nimmt den Niederschlag aus sauerer Lösung anf.

Das Product in alkoholischer Lösung gibt mit alkoholischer Platinchloridlösung einen krystallinischen, auffallend lichtgelb gefärbten Niederschlag.

In Oxalsäure ebenso wie in Weinsäure löst sich das Product, beim Erwärmen tritt jedoch Trübung, Ölabscheidung und starker Geruch nach Methylaethylacroleïn auf.

Zur Elementaranalyse verwandte ich:

I. $0 \cdot 2400 g$ Substanz, welche $0 \cdot 6056 g$ Kohlensäure und $0 \cdot 2472 g$ Wasser lieferten.

II. $0 \cdot 3536 g$ Substanz, welche $0 \cdot 8920 g$ Kohlensäure und $0 \cdot 3643 \mathrm{~g}$ Wasser lieferten.

III. Bei der Stickstoff bestimmung nach Dumas lieferten $0.2217 g$ Substanz $0 \cdot 04097 g$ Stickstoff. 
Auf 100 Theile:

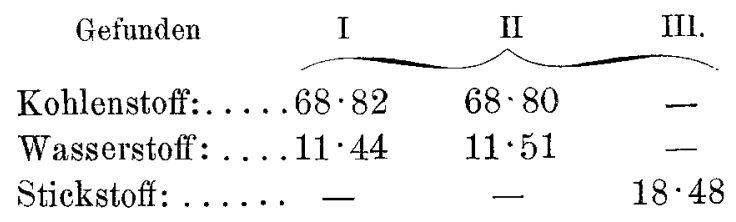

Wenn auch unter der Annahme, dass die analysirten Substanzen nicht ganz rein gewesen seien, vielleicht eine oder die andere Formel, wie $z$. B.:

$\begin{array}{ll}\underbrace{\mathrm{C}_{12} \mathrm{H}_{25} \mathrm{~N}_{3}}_{\text {Koblenstoff: } \ldots 68 \cdot 25} & \underbrace{\mathrm{C}_{18} \mathrm{H}_{36} \mathrm{~N}_{4}}_{78} \\ \text { Wasserstoff: } .11 \cdot 85 & 11 \cdot 69 \\ \text { Stickstoff: } \ldots .19 \cdot 90 & 18 \cdot 19\end{array}$

sich berechnen liesse, so ist doch keine Gewähr dafür vorhanden, dass das analysirte Product ein einheitliches ist. Ich trachtete daher durch fractionirte Fällung eine Trennung zu bewirken, die vielleicht zur Gewinnung eines einheitlichen Körpers fübren konnte. Allein weder die Anwendung von alkoholischem Platinchlorid, obwohl krystallinisehe Niederschläge erbalten wurden, noch die von Kaliumquecksilberjodid als Fällungsmittel gestatteten mir, dieses Ziel zu erreichen. Aus den durch letzteres erhaltenen Niederschlägen konnte man zur ursprïnglichen Base nicht mehr zurïckgelangen.

Ich musste daher die Untersuchung dieses bei $100^{\circ}$ erhaltenen Einwirkungsproductes bei Seite lassen und wende mich jenen Producten zu, die ich erhielt durch die

\section{Einwirkung von Ammoniak auf Methylaethylacroleïn bei $200^{\circ} \mathrm{C}$.}

Schliesst man das vom Alkohol befreite Rohproduct neuerdings in Rohre ein und erhitzt diese bis zu $200^{\circ} \mathrm{C}$., so tritt eine tiefgehende Zersetzung desselben ein.

Nimmt man nämlich nach 12 Stunden die Rohre aus dem Ofen, so zeigt es sich beim Öffnen, dass ein bedeutender Druck vorhanden ist, und zwar entweicht Ammoniak, welches offenbar 
bei der Veränderung, die durch die Erwärmung vor sich gegangen ist, abgespalten wurde. Die Rohre wurden nun neuerdings eingelegt, nach mehrstündiger Erhitzung auf $200^{\circ}$ wieder geöffnet, und dieses Verfahren solange fortgesetzt, bis beim Öffnen kein Druck, das ist kein Ammoniakgas mehr vorhanden war; in Ganzen erhitzte ich 40 bis 42 Stunden.

Der Röhreninhalt wurde in Salzsäure gelöst und mit Äther ausgeschüttelt, der $\ddot{A}$ ther färbte sich violett-roth und.hinterliess beim Verdunsten eine ebenso gefärbte, ölige, schmierige Flïssigkeit von angenehmem Geruche, der an den der kohlenstoffreicheren Alkohole erinnert. Destillirt man diesen Rückstand, so erhält man eine hellgelbe, auf Wasser schwimmende, klare Flüssigkeit, welche nach Condensationsproduct riecht. Durch Sehütteln mit Natriumbisulfit wird die Substanz vom begleitenden Methylaethylacroleïn befreit und verliert gleichzeitig die gelbe Färbung; mit Wasser gewaschen und mitPotasche getrocknet, ging der grösste Theil bei der Destillation zwischen 150 und $170^{\circ}$ über. Die geringe Menge dieses so erhaltenen Alkohols machte es unmöglich, den Siedepunkt oder direct die Zusammensetzung zu bestimmen. Ich verwandelte ihn daher durch Erhitzen mit überschïssigem Essigsäureanhydrid in der von Lieben und $\mathrm{Z}$ e ise ${ }^{1}$ angegebenen Weise in das Acetat. Dieses gewaschen und getrocknet ging bei der Destillation zwischen 155 und $165^{\circ} \mathrm{C}$. beinahe vollständig über.

Die Analyse ergab die folgenden Zablen:

$0 \cdot 2198 \mathrm{~g}$ Substanz lieferten bei der Verbrennung $0.5339 \mathrm{~g}$ Kohlensäure und $0 \cdot 2032 g$ Wasser.

Dies entspricht $66 \cdot 24 \%$ Kohlenstoff und $10 \cdot 27 \%$ Wasserstoff, während sich fur Hexylacetat $66 \cdot 67 \%$ Kohlenstoff und $11 \cdot 11 \%$ Wasserstoff berechnen.

Bei der iberaus geringen Menge der vorhandenen Substanz war es unmöglich gewesen, den Alkohol mehrfach durchzufractioniren und zu reinigen, und es mag dies die geringere Übereinstimmung der Zahlen vielleicht erklären. Jedenfalls ist es wahrscheinlich, aber nicht sicher, dass die vorgelegene Sub-

1 Monatsh. f. Chemie IV., 31. 
stanz Hexylalkohol gewesen sei, der durch Reduction aus $\mathrm{C}_{6} \mathrm{H}_{10} \mathrm{O}$ entstanden wäre.

Die salzsauere Lösung des auf $200^{\circ}$ erhitzten basischen Productes wurde mit Ätzkali wieder alkalisch gemacht, und die sich ölig in brauner Farbe abscheidenden Basen mit Wasserdampf übergetrieben, in vorgelegten Rölren aufgefangen, und die sich über dem Wasser jetzt klar abscheidende Ölschichte abgehoben.

Da aber die Substanz etwas in Wasser löslich ist und auch gern Emulsionen bildet, wurden die wässerigen Destillate noch mehrmals destillirt und auf diese Weise noch weitere Substanz gewonnen. Zur Trocknung verwandte ich Ätzkali, jedoch gelang es nicht, dadurch vollkommen trockene Substanz zu erhalten, weshalb ich bei den späteren Destillationen und Fractionirungen metallisches Natrium zum Trocknen anwandte.

Nach 20- bis 25maligem Fractioniren hatte ich von dem Producte, welches ich durch Erhitzen auf $200^{\circ}$ gewonnen hatte, eine Reihe von 14 Fractionen zwischen 165 und $260^{\circ}$ erhalten, von welchen, mit Ausnahme zweier, alle ziemlich gleich gross sind. Nur die Fractionen zwischen 195 und $198^{\circ}$ und zwischen 230 und $235^{\circ}$ sind jede beilänfig 8 - bis $10 \mathrm{mal}$ so gross als die übrigen Zwischenfractionen, so dass man sagen kann, dass die aus dem Rohproduct durch Erhitzen auf $200^{\circ}$ gewonnenen Körper zu einem Drittheil aus dem zwischen 195 und $198^{\circ}$ siedenden und zu einem Drittheil aus dem zwischen 230 und $235^{\circ}$ siedenden basischen Körpern bestehen, während das letzte Drittel durch die Vor-, Nach- und Zwischenfractionen und die Manipulationsverluste aufgezehrt wird.

Der Destillationsrückstand ist sehr gering, tiefbraun bis schwarz gefärbt, dick, harzig.

Die Fraction 165 bis $175^{\circ}$ soll später besprochen werden.

Die Fraction 195 bis $198^{\circ}$ erwies sich als ein

\section{Parvolin.}

Die Base bildet eine klare, wasserhelle, leicht bewegliche, lichtbrechende Flüssigkeit, die nach längerem Stehen einen Stich ins Gelbe annimmt; sie hat einen starkev, dem Koniin 
E. Hoppe,

nicht ganz unähnlichen, widerlichen Geruch, sehr bitteren Geschmack, ist im Wasser weniger als in Alkohol und Äther löslich und reagirt stark alkalisch.

Die schwach salzsauere Lösung der Base gibt mit:

Platinchlorid: krystallinischen Niederschlag;

Goldchlorid: ölige Ausscheidung;

Quecksilberchlorid: ölige Ausscheidung;

Pikrinsäure: krystallinischen Niederschlag;

Cadmiumjodid: ölige Ausscheidung;

Cadminmchlorid: keinen Niederschlag;

Jodjodkalium: ölige Ausscheidung;

Kaliumquecksilberjodid: ölige Ausscheidung, gelb erstarrend;

Phosphorwolframsäure: weissen, amphoren Niederschlag;

Phosphormolybdänsäure: strohgelben, amphoren Niederschlag.

Die Analyse der sorgfältig getrockneten Base ergab die folgenden Zahlen:

I. $0 \cdot 2334 g$ Substanz lieferten bei der Verbrennung $0.6831 \mathrm{~g}$ Kohlensäure and $0.2000 \mathrm{~g}$ Wasser.

II. $0 \cdot 2700 g$ Substanz lieferten bei der Verbrennung $0 \cdot 7875 g$ Kohlensäure und $0 \cdot 2289 g$ Wasser.

III. $0 \cdot 2548 g$ Substanz lieferten bei der Stickstoff bestimmung nach D umas $0.02577 g$ Stickstoff.

IV. $0 \cdot 1940 g$ Substanz lieferten bei der Stickstoff bestimmung nach Dumas $0 \cdot 02117 g$ Stickstoff.

In 100 Theilen:

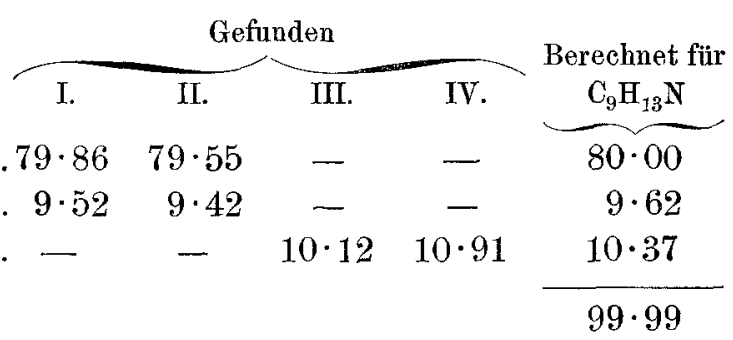

Das Platindoppelsalz der Base erhielt ich nach mehrfachem Umkrystallisiren in orangegelben, durchscheinenden, 2 bis $3 \mathrm{~mm}$ 
langen Krystallen, deren Schmelzpunkt bei $188^{\circ}$ (uncorr.) gefunden wurde. Es ist in Alkohol und warmem Wasser leicht löslich.

Herr Dr. Rudolf Köchlin hatte die Güte, die Messung derselben vorzunehmen und theilte mir folgendes Resultat mit:

Die Substanz ist monosymmetrisch.

Axenverhältniss $\mathrm{a}: \mathrm{b}: \mathrm{c}=0 \cdot 4142: 1: 0 \cdot 4979$

$$
\beta=63^{\circ} 8^{\prime} 47^{\prime \prime} \text {. }
$$

Beobachtete Formen: (010), (110), (011), (101), (111), (121).

Winkel:

\begin{tabular}{|c|c|c|}
\hline Fläche & Gerechnet & Gemessen \\
\hline$(010):(110)$ & - & $67^{\circ} 30^{\prime}$ \\
\hline$(010):(011)$ & - & $63^{\circ} 32^{\prime}$ \\
\hline$(110):(011)$ & 一 & $57^{\circ} \quad 2^{\prime}$ \\
\hline$(1 \overline{1} 0):(011)$ & $78^{\circ} 17^{\prime} 5^{\prime \prime}$ & $78^{\circ} 20^{\prime}$ \\
\hline$(100):(\overline{1} 01)$ & $130^{\circ} \quad 3^{\prime} 42^{\prime \prime}$ & - \\
\hline
\end{tabular}

Kleinere Krystalle verwandte ich zur Elementaranalyse:

I. $0 \cdot 2013 g$ Substanz lieferten $0 \cdot 2341 g$ Kohlensäure and $0.0783 g$ Wasser.

II. $0 \cdot 2073 g$ Substanz gaben beim Glühen $0 \cdot 0594 g$ Platin. In 100 Theilen:

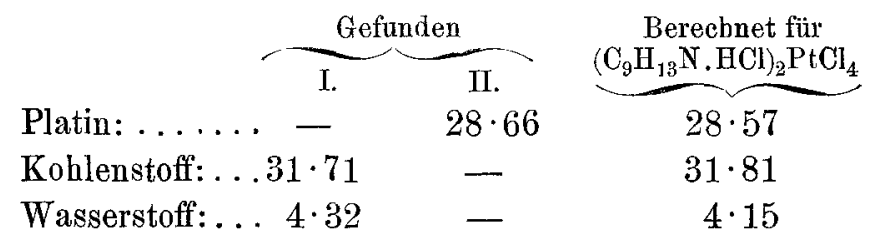

Die gefundenen Procentzahlen stehen also in guter Übereinstimmung mit jenen, welche die Formel

$$
\left(\mathrm{C}_{9} \mathrm{H}_{13} \mathrm{~N} . \mathrm{HCl}\right)_{2} \mathrm{PtCl}_{4}
$$

beansprucht, die Procentzahlen der Analyse der freien Base nähern sich ebenfalls der Formel:

$$
\mathrm{C}_{9} \mathrm{H}_{13} \mathrm{~N}
$$

welche dem Parvolin zukömmt. 
Es scheint das vorliegende Parvolin verschieden zu sein von dem von Öchsner de Coninck ${ }^{1}$ aus Cinchonin und Ätzkali erhaltenen bei $220^{\circ}$ siedenden, verschieden auch von dem von Dürkopf and Schlaugk ${ }^{2}$ aus Propionaldehydammoniak und Paraldehyd erhaltenen bei $188^{\circ}$ siedenden Parvolin, das einen mild angenehm süsslichen Geruch besitzt und sich besonders in der Oxydation unterscheidet. Möglicherweise ist es zu identificiren mit dem von Williams ${ }^{3}$ aus bituminösem Schiefer dargestellten, bei $188^{\circ} \mathrm{C}$. siedenden und dem von Thenius ${ }^{4}$ aus dem Steinkohlentheer gewonnenen Parvolin. Sicher aber ist das vorliegende Parvolin als identisch anzusehen mit dem von W aage ${ }^{5}$ durch Einwirktng von Ammoniak auf Propionaldehyd dargestellten, indem es ja von vornherein zu erwarten ist, dass aus Propionaldehyd und dem Condensationsproducte desselben durch Einwirkung desselben Körpers auch dieselben Substanzen entstehen. Ausserdem ist der Siedepunkt, den Waage mit 198 bis $200^{\circ} \mathrm{C}$. angibt, der Schmelzpunkt des Platindoppelsalzes bei $188^{\circ}$, die Löslichkeit in Wasser, Alkohol und $\ddot{A}$ ther, die Reactionen mit Pikrinsäure, Kaliumquecksilberjodid, Phosphorwolframsäure analog auch von mir gefunden worden. Es spricht ferner für die Identität noch die

\section{Oxydation des Parvolin.}

Zur Oxydation verwandte ich $7 \frac{1}{2} g$ Parvolin von der analysirten Fraction vom Siedepunkte 195 bis $198^{\circ}$, welche ich nach Waage in Wasser zu einer Emulsion vertheilte und am Rückflusskühler unter langsamem Zusatz von $72 \mathrm{~g}$ Kaliumpermanganat in vierprocentiger Lösung vier Tage lang erwärmte. Nach der Entfernung des Braunstein wurde die Lösung eingeengt, mit Schwefelsäure neutralisirt und das Cadmiumsalz dargestellt.

1 Compt. rend. XCI., 296.

2 Berl. Ber. 21, 832.

3 Jahresber. 1854, 405 und Journ. f. prakt. Chem. 62, 467.

4 Jahresber. 1861, 502.

5 Monatsh. f. Chemie IV, 721 . 
Das bei $130^{\circ}$ getrocknete Salz wurde der Analyse unterzogen:

I. $0 \cdot 1725 g$ Substanz gaben bei der Zerlegung mit Schwefelwasserstoff $0 \cdot 0802 g$ Cadmiumsulfid.

II. $0 \cdot 2223 g$ Substanz gaben bei der Verbrennung $0 \cdot 2182 g$ Kohlensäure und $0.0462 g$ Wasser.

In 100 Theilen:

\begin{tabular}{|c|c|c|c|}
\hline & \multicolumn{2}{|c|}{ Gefunden } & Bereehnet für \\
\hline & I. & II. & $\mathrm{C}_{7} \mathrm{H}_{3} \mathrm{NO}_{4} \cdot \mathrm{Cd}+2 \mathrm{H}_{2} \mathrm{O}$ \\
\hline Cadmium: . & $.36 \cdot 17$ & - & $35 \cdot 79$ \\
\hline Kohlenstoff:. & - & $26 \cdot 77$ & $26 \cdot 84$ \\
\hline Wasserstoff:. & - & $2 \cdot 31$ & $2 \cdot 23$ \\
\hline
\end{tabular}

Wenn diese Zahlen auch in guter Übereinstimmnung mit den für das Cadmiumsalz der Pyridindicarbonsäure geforderten stehen, so gelang es mir leider nicht, dieselbe Übereinstimmung. für die freie Säure zu erlangen. Um dieselbe darzustellen, zerlegte ich das Cadmiumsalz unter heissem, essigsäurehältigem Wasser mit Schwefelwasserstoff. Durch Eindampfen der vom Cadmiumsulfid getrennten Lösung erhielt ich die freie Säure, sie musste öfters aus heissem Wasser umkrystallisirt werden, bis der Geruch nach Essigsäure schwand und die mittleren Fractionen weiss wurden.

Die freie Säure krystallisirt in mikroskopischen Nadeln, sie ist in kaltem Wasser fast gar nicht, in heissem Wasser sebwer lösbar, einmal gelöst, fällt sie aber beim Erkalten der Lösung nur dann aus, wenn diese weiter concentrirt wurde. In heissem Alkohol ist sie schwer, in Äther gar nicht löslich.

Mit Eisenvitriol gibt die Säure eine rothgelbe Farbenreaction, fällt jedoch bald einen braunen Niederschlag. Mit Bleizucker gibt sie weisse Flocken. Silbernitrat bringt einen flockigen, weissen Niederschlag hervor, Kupferacetat einen blauen Niederschlag, der aufs Filter gebracht einen Stich ins Violette zeigt. Den Schmelzpunkt fand ich bei $223^{\circ} \mathrm{C}$. an einem Thermometer, das durch eine Schmelzpunktbestimmung des Anthracen controlirt war. 
Die Analysen ergaben einen etwas zu hohen Gehalt an Kohlenstoff and Wasserstoff. Leider gestattete mir jedoch die geringe Menge meiner Substanz nicht, weitere Reinigungsversuche anzustellen und diesen Mangel an Übereinstimmung aufzuklären. Ich kann nur muthmassen, dass entweder theilweise eine Aufnahme von Essigsäure, die ich als Lösungsmittel des Cadmiumsalzes angewandt hatte, stattgefunden, oder dass bereits durch das oftmalige Erhitzen und Eindampfen zurTrockne eine theilweise Umsetzung in die Monocarbonsäure vor sich gegangen war.

Von den sechś möglichen Dicarbonsäuren war durch die rothe Eisenreaction, sowie durch den niedrigen Schmelzpunkt die $\beta \beta$-Diearbonsäure (Hautzseh and L. Weiss) und die By-Säure oder Cinchomeronsäure von vornherein ausgeschlossen.

Der Schmelzpunkt konnte zur weiteren Unterscheidung nicht herangezogen werden, weil einerseits die vier übrig gebliebenen Dicarbonsäuren ziemlich nahe aneinanderliegende Schmelzpunkte besitzen, anderseits bei jeder einzelnen die Angaben der versehiedenen Darsteller differiren.

Während Hoogewerff und van Dor ${ }^{1}$ den Schmelzpunkt der Chinolinsäure mit 222 bis $225^{\circ}$ angeben, fand ihn Skraup ${ }^{2}$ bei $231^{\circ}$.

Den Schmelzpunkt der Isocinchomeronsäure geben Weide 1 und Herzig ${ }^{3}$ mit $236^{\circ}$, Ramsay und Dobbie ${ }^{4}$ mit $237 \cdot 5^{\circ}$ an, Lunge und Rosenberg ${ }^{5}$ fanden ihn bei $230^{\circ}$.

Die von Ladenburg und $R_{0} \operatorname{th}^{6}$ dargestellte $\alpha \alpha^{\prime}$-Dicarbonsäure schmilzt bei $226^{\circ}$ und soll - wie dieselben Herren behaupten - identisch sein mit der von Dewar gefundenen bei $210^{\circ}$ schmelzenden Säure.

Die Lutidinsäure, deren Schmelzpunkt Weidel und Herzig ${ }^{8}$ mit $219^{\circ}$ angaben, wurde von Böttinger ${ }^{9}$ mit

1 Berl. Ber. 12, 748.

2 Monatsh, f. Chemie II, 148.

3 Monatsh. f. Chemie I, 5.

4 Berl. Ber. 11, 325.

5 Berl. Ber. 20, 135.

6 Berl. Ber. 18. 53.

7 Zeitsch. f. Chem. 1871, 116.

8 Monatsh. f. Chemie I, 20.

9 Berl. Ber. 17, 93. 
234 bis $235^{\circ}$, von Laderburg und Roth ${ }^{1}$ bei $235^{\circ}$ schmelzend gefunden, und die letzteren geben an, dass auch sie bei weniger sorgfältiger Darstellung eine bei $220^{\circ}$ schmelzende Lutidinsäure erhalten haben; auch Lunge und Rosenberg ${ }^{2}$ fanden zwei Schmelzpunkte bei $223^{\circ}$ und bei $235^{\circ}$.

Ähnlich differiren die bisher vorliegenden Angaben über den Krystallwassergehalt der Säuren selbst und ihrer Salze, so dass es am sichersten erschien, den Zerfall in die Monocarbonsäure vorzunehmen und letztere zu untersuchen, umsomehr als die Analysenziffern meiner freien Dicarbonsäure mangelhafte gewesen waren.

\section{Monocarbonsäure.}

Die Darstellung derselben warde nach der von WeideI und Herzig ${ }^{3}$ angegebenen Methode in einer kleinen Retorte durch Erhitzen im Wasserstoffstrome anf $250^{\circ}$ ausgeführt. In vorgelegtem Barytwasser wurde die Kohlensätrereaction beobachtet, auch war intensiver Pyridingeruch bemerkbar.

Das Sublimationsproduct war stellenweise weiss, in kleinen schönen Krystallen, welche ich sofort zur Schmelrpunktbestimmung verwandte, ich fand diesen bei $222^{\circ}$ (corr. mittelst Anthracen). Die weniger schönen, gelblichen Sublimationsproducte einerseits, der schwarze Rückstand in der Retorte anderseits wurden gesondert mit Ammoniak aufgenommen und mit Kupferacetat in das Kupfersalz verwandelt, dasselbe ist schön lichtblau und in Wasser nicht löslich.

Das Kupfersalz wurde mit Schwefelwasserstoff zerlegt, das Filtrat vom Schwefelknpfer mit Thierkohle entfärbt und bis zur Trockne eingeengt.

Die Säure scheidet sich aus concentrirter, heisser Lösung beim Erkalten in kleinen, feinen Nadeln, beim Eindampfen zur Trockene pulverförmig aus und schmilzt übereinstimmend mit dem Sublimat bei $222^{\circ}$. Krystallwasser fand ich keines.

1 Berl. Ber. 18, 916.

2 Berl. Ber. 20, 132.

3 Monatsh. f. Chemie I., 16. 
Die Elementaranalyse ergab:

$0 \cdot 1261 g$ Substanz lieferten $0 \cdot 2665 g$ Kohlensäure, $0 \cdot 0470 g$ Wasser und hinterliessen $0 \cdot 0018 g$ unverbrannten Rückstand im Schiffchen.

In Procenten:

Kohlenstoff: . . .58.49
Wasserstoff: . . $4 \cdot 20$$\quad \begin{array}{r}\begin{array}{c}\text { Berechnet für } \\ \mathrm{C}_{6} \mathrm{H}_{5} \mathrm{NO}_{2}\end{array} \\ 58 \cdot 53 \\ 4 \cdot 06\end{array}$

Das Silbersalz der Monocarbonsäure wird durch Silbernitrat aus der neutralen Lösung des Ammoninmsalzes weiss gefällt und lieferte bei der Verbrennung die folgenden Zahlen:

$0.0850 \mathrm{~g}$ Substanz gaben $0.0982 g$ Kohlensäure, $0.0125 g$ Wasser und hinterliessen $0.0396 g$ Silber als Rückstand im Schiffehen.

In Procenten:

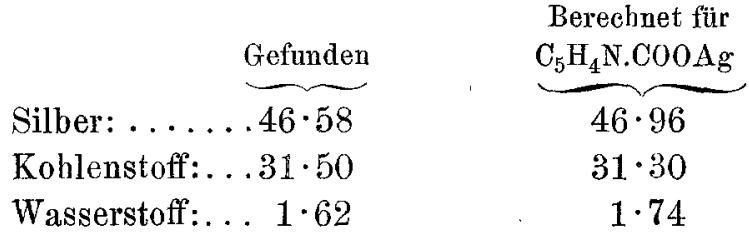

Die Analysenresultate der Säure und ihres Silbersalzes stehen in guter Übereinstimmung mit den Procentzahlen der berechneten Formeln, so dass man füglich auf eine Pyridinmonocarbonsäure schliessen darf. Der Schmelzpunkt der allerdings trotz oftmaligen Umkrystallisirens noch immer nicht ganz reinen Säure, im Vereine mit den Eigenschaften des Silber- und Kupfersalzes, weist auf Nicotinsäure hin.

Es zeigt sich dadurch, dass die Vermuthung Wagge's, es mit Lutidinsäure zu thun gebabt zu haben, unbestätigt bleibt, und dass die vorgelegene Dicarbonsäure mit der Isocinchomeronsäure zu identificiren ist.

Die schwerere Löslichkeit, das blauviolette Kupfersalz, das flockige Silbersalz schliessen die Chinolinsäure aus, während gegen die Isocinchomeronsäure nur das Eine angeführt werden könnte, dass sie vielfach mit Krystallwasser gefunden worden 
ist; doch gibt es auch Gegenangaben, so fanden Ramsay ${ }^{1}$ und Eppstein ${ }^{2}$, dass die aus heisser Lösung ausgeschiedenen, nicht in der Mutterlauge belassenen Krystalle kein Krystallwasser enthalten.

Ich glaube daher das aus Methylaethylacroleïn durch Einwirkung von Ammoniak erhaltene Parvolin als ein Homologes des Pyridin ansehen zu dürfen, welches in der $\alpha$ - und $\beta^{\prime}$-Stellung Seitenketten führt.

\section{Fraction 230 bis $235^{\circ}$.}

Die Base bildet eine klare, wasserhelle, noch immer bewegliche, schwach blau fluorescirende Flüssigkeit, die nach längerem Stehen einen Stich ins Gelbe annimmt, ihr Geruch ist weniger intensiv, aber sehr ähnlich dem des Parvolin, der Geschmack bitter. In Wasser ist sie wenig löslich, wohl aber in Alkohol und $\ddot{\mathrm{A}}$ ther.

Die schwach salzsanere Lösung der Base gibt mit:

Platinchlorid: öligen, später krystallisirenden Niederschlag;

Goldchlorid: öligen, später krystallisirenden Niederschlag;

Quecksilberchlorid: ölige Ausscheidung;

Pikrinsäure: ölige Ausscheidung;

Cadmiumjodid: ölige Ausscheidung:

Cadmiumchlorid: ölige Ausscheidung;

Jodjodkalium: ölige Ausscheidung;

Kaliumquecksilberjodid: ölige Ausscheidung;

Phosphorwolframsäure: keine Atusscheidung;

Phosphormolybdänsäure: amorphen gelben Niederschlag.

Die Analyse der sorgfältig getrockneten Base ergab die folgenden Zahlen:

I. $0 \cdot 3232 g$ Substanz lieferten bei der Verbremung $0 \cdot 9585 g$ Kohlensäure und $0 \cdot 3038 g$ Wasser.

II. $0 \cdot 2315 \mathrm{~g}$ Substanz lieferten bei der Verbrennung $0 \cdot 6848 g$ Kohlensäure und $0 \cdot 2105 \mathrm{~g}$ Wasser.

III. $0 \cdot 1983 \mathrm{~g}$ Substanz lieferten bei der Stickstoffbestimmung nach Dumas $0 \cdot 01615 g$ Stickstoff.

1 Jahresber. 1878, 438.

2 Ann. 231, 26. 
In 100 Theilen :

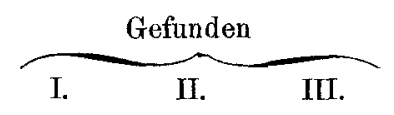

Kohlenstoff $\ldots 80 \cdot 87 \quad 80 \cdot 67 \quad \ldots$

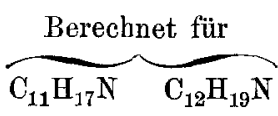

$80 \cdot 98 \quad 82 \cdot 51$

$10 \cdot 43 \quad 10 \cdot 74$

Die Dampfdichte bestimmte ich nach dem von Goldschmiedt und Ciamician ${ }^{1}$ angegebenen Verfahren.

I. Gewicht der angewandten Substanz: $0.0429 \mathrm{~g}$.

Gewicht des angewandten Quecksilbers: $618 \cdot 45 \mathrm{~g}$.

Gewicht des ausgeflossenen Quecksilbers: $192 \cdot 12 \mathrm{~g}$.

Anfangstemperatur des Quecksilbers: $17^{\circ} \mathrm{C}$.

Temperatur des Metallbades: $271^{\circ} \mathrm{C}$.

A bgemessene Höhe der wirksamen Quecksilbersäule: $77 \mathrm{~mm}$.

Barometerstand, reducirt auf $0^{\circ}: 757.5 \mathrm{~mm}$.

Specifisches Gewicht des Quecksilbers bei $0^{\circ}$ C.: $13 \cdot 596 \mathrm{~g}$.

Tension des Quecksilberdampfes bei $270^{\circ}: 126 \cdot 22$

II. Gewicht der angewandten Substanz: $0.0334 \mathrm{~g}$.

Gewicht des angewandten Quecksilbers: $621 \cdot 3 \mathrm{~g}$.

Gewicht des ausgeflossenen Quecksilbers: $166 \cdot 2 \mathrm{~g}$.

Anfangstemperatur des Quecksilbers: $17^{\circ} \mathrm{C}$.

Temperatur des Metallbades: $287^{\circ} \mathrm{C}$.

Ảogemessene Höhe der wirksamen Quecksilbersäule: $82 \mathrm{~mm}$.

Barometerstand, reducirt auf $0^{\circ}: 747.5 \mathrm{~mm}$.

Specifisches Gewicht des Quecksilbers bei $0^{\circ}$ C.: $13 \cdot 596$

Tension des Quecksilberdampfes bei $287^{\circ}: 182 \cdot 67$

Daraus ergab sich die Dampfdichte:

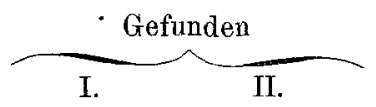

Dampfdichte .... $5 \cdot 465 \quad 5 \cdot 714$

Moleculargewicht $.157 \cdot 9 \quad 165 \cdot 2$

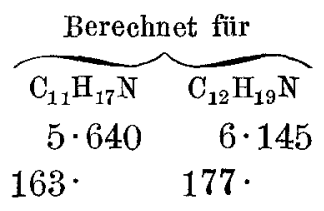

177 .

Die empirische Formel für die untersuchte Substanz scheint demnach $\mathrm{C}_{11} \mathrm{H}_{17} \mathrm{~N} z u$ sein. Die nachfolgende Untersuchung des

I Berl. Ber. 10, 641. 
Platin- and Goldsalzes zeigte jedoch, dass ein Gemisch eines Körpers $\mathrm{C}_{12} \mathrm{H}_{19} \mathrm{~N}$ mit einem kohlenstoffreicheren und einem kohlenstoffärmeren - wahrscheinlich Parvolin - vorgelegen war.

Eine fractionirte Fällung der salzsauren Lösung der Base mit Platinchlorid lieferte Fractionen, welche deutlich zeigten, dass ein Gemenge vorhanden sei, gestatteten aber nicht, eine vollkommene Trennung durchzuführen.

Besser gelang dies durch eine fractionirte Fällung mit Quecksilberchlorid: $10 \cdot 3 g$ der Base ${ }^{1}$ wurden mit je $2 \cdot 2 g$ Quecksilberchlorid in vier Fractionen gefällt und ergaben an Grösse abnehmende, durchaus ölige Ausscheidungen, jedesmal wurden dieselben sofort am Filter, nachdem sie gewaschen waren, mit Alkohol - worin sie leicht löslich sind - aufgenommen und mit Schwefelwasserstoff rom Quecksilber befreit. Aus der filtrirten, mit Chlorwasserstoffsäure angesäuerten und dann eingeengten Lösung wurde die Base mit Ätzkali wieder freigemacht, mit Wasserdampf überdestillirt und sofort in das Chloroplatinat erwand elt. Nachdem die vierte Fraction des Chlorquecksilberdoppelsalzes ausgefallen war, wurde das Filtrat desselben sammt den Waschwässern destillirt. Das Destillat hinterliess nach dem Einengen keinerlei Rückstand. Im Destillationskolben aber schied sich beim Erkalten eine fünfte, ölige, bedeutende Fraction des Quecksilberchloridsalzes aus, welche auf die gleiche Weise wie die ersten vier behandelt und in das Platindoppelsalz übergeführt wurde.

Die vierte und fünfte Fraction als Platinsalze zeigen denselben Schmelzpunkt $135^{\circ}$ (uncorr.). Das Salz hat die bei den Platindoppelsalzen gewöhnlich beobachtete orangegelbe Farbe, krystallisirt, ist in Wasser wenig, in Alkohol leicht löslich.

Herr Dr. Rudolf Köchlin hatte die Güte, die Krystalle zu messen und theilt mir hieruber Folgendes mit:

„Die Krystalle sind monoklin.

Axenverhältniss: $\mathrm{a}: \mathrm{b}: \mathrm{c}=1 \cdot 1166: 1: 1 \cdot 1078$

$$
\beta=73^{\circ} 20^{\prime} 50^{\prime \prime} \text {. }
$$

1 Von einer zweiten Darstellung. Die Base war diesmal noch öfter fractionirt destillirt worden, wodurch der kohlenstoffärmere Körper (Parvolin) entfernt worden war. 
Die auftretenden Formen sind:

$$
\begin{aligned}
& \text { ein Prisma.... .m }=(110) \\
& \text { ein Klinodoma. . } d=(011)
\end{aligned}
$$

ausserdem tritt manchmal noch ein zweites Klinodoma (094), aber stets nur auf einer Seite der Symmetrie-Ebene auf.

Die Winkel sind:

\begin{tabular}{|c|c|c|c|}
\hline Zeichen & Symbole & \multirow{2}{*}{ Gemessen } & Gerechnet \\
\hline \multicolumn{2}{|c|}{ der Flächen } & & \\
\hline$m_{1}: m_{2}$ & $(110:(1 \overline{1} 0)$ & $98^{\circ} 47^{\prime}$ & - \\
$m_{1}: d_{1}$ & $(110):(011)$ & $46^{\circ} 53^{\prime}$ & - \\
$d_{1}: d_{2}$ & $(011):(0 \overline{1} 1)$ & $94^{\circ} 19^{\prime}$ & - \\
$d_{1}: m_{1}$ & $(011):(\overline{1} 10)$ & $64^{\circ} 33^{\prime}$ & $64^{\circ} 32^{\prime} 40^{\prime \prime}$ \\
\hline
\end{tabular}

Die Krystalle haben zwar glänzende Flächen, doch geben dieselben keine guten Reflexe; Flächenkrümmungen, $\mathrm{Ab}$ weichungen vom Flächenparallelismus, überdies der Umstand, dass die Krystalle an der Luft sich sehr schnell zersetzen, ersehweren eine genaue Messung, und sind die Resultate deshalb nicht als völlig sichere zu bezeichnen."

Die Elementaranalyse der fünften Fraction ergab die folgenden Resultate:

I. $0 \cdot 2080 g$ Substanz lieferten $0 \cdot 2857 g$ Kohlensäure, $0 \cdot 1006 g$ Wasser und hinterliessen am Schiffehen $0.0528 g$ Platin ${ }^{1}$.

II. $0 \cdot 2018 g$ Substanz lieferten $0 \cdot 2762 g$ Kohlensäure, $0 \cdot 0940 g$ Wasser und hinterliessen am Schiffchen $0.0510 \mathrm{~g}$ Platin. In 100 Theilen:

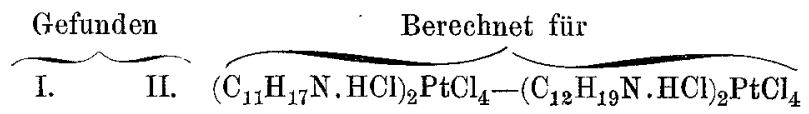
Platin: . . 25 $25925 \cdot 27$ $26 \cdot 43$
$25 \cdot 45$
Kohlenstoff: $37 \cdot 46 \quad 37 \cdot 33$
$35 \cdot 88$
$37 \cdot 72$
Wasserstoff: $5 \cdot 36 \quad 5 \cdot 17$
$4 \cdot 89$
$5 \cdot 24$

1 Um sicher zu gehen, wurde das Schiffehen erst in ein $12 \mathrm{~cm}$ langes Platinrohr, welches ebenfalls gewogen war, gegeben, und in diesem in das Verbrennungsrohr eingeführt, so dass kein Platin im Verbrennungsrohre verstäubt zurückgeblieben sein kann. 
Die ersten drei Fractionen gaben mehr Kohlenstoff und dementsprechend weniger Platin.

Um eine weitere Bestätigung für die Richtigkeit dieser Analysen zu besitzen, wurde das Platinsalz mit Schwefelwasserstoff zerlegt und die verbleibende salzsaure Lösung der Base mit Goldchlorid versetzt. Es scheidet sich ein tiefgelber, öliger Niederschlag aus, der gut gewaschen ins Vacuum gestellt wurde, wo er alsbald zu einer schön goldgelben, krystallinischen, weichen, leicht zerreibbaren Masse erstarrte. Den Sehmelzpunkt derselben beobachtete ich bei $93^{\circ} \mathrm{C}$. (uncorr.), jedoch beginnt die Substanz bereits bei $75^{\circ} \mathrm{zu}$ sintern.

Die Analyse dieses aus der vierten und fiunften Fraction der Quecksilberchloridfällung stammenden Goldsalzes ergab die folgenden Zahlen:

I. $0.2127 g$ Substanz lieferten beim Glühen $0.0806 g$ Gold.

II. $0 \cdot 2195 g$ Substanz lieferten bei der Verbrennung $0 \cdot 2220 g$ Kohlensäure, $0 \cdot 0685 g$ Wasser und hinterliessen als Rückstand am Schiffehen $0 \cdot 0833 g$ Gold.

III. $0.2257 g$ Substanz lieferten bei der Verbrennung $0.2268 g$ Kohlensäure, $0 \cdot 0715 g$ Wasser und hinterliessen als Rückstand am Schiffchen $0.0852 g$ Gold.

In 100 Theilen:

\begin{tabular}{|c|c|c|c|c|}
\hline & \multicolumn{3}{|c|}{ Gefunden } & \multirow{2}{*}{$\begin{array}{c}\text { Berechnet für } \\
\mathrm{C}_{12} \mathrm{H}_{19} \mathrm{~N} . \mathrm{HCl} . \mathrm{AuCl}\end{array}$} \\
\hline & I. & II. & III. & \\
\hline Gold: . & $.37 \cdot 89$ & $37 \cdot 95$ & $37 \cdot 75$ & $37 \cdot 98$ \\
\hline Kohlenstoff: & - & $27 \cdot 59$ & $27 \cdot 40$ & $27 \cdot 90$ \\
\hline Wasserstoff; & . - & $3 \cdot 47$ & $3 \cdot 52$ & $3 \cdot 87$ \\
\hline
\end{tabular}

Leider war es mir wegen Mangel an Substanz nicht möglich, die freie Base, durch das Quecksilberchloridsalz gereinigt, wieder in freiem Zustande zu analysiren, weil die Ausbeute hiedurch zu gering wird, als dass eine Trocknung und mehrfache Destillation der Base ausführbar wäre. Trotzdem aber glaube ich, dass die durch die Salze gegebene Formel:

$$
\mathrm{C}_{12} \mathrm{H}_{19} \mathrm{~N}
$$

die richtige sein dürfte, weil die Nebenfractionen wirklich kohlenstoffreichere und kohlenstoffärmere Körper enthalten, und 
besonders weil die Base aus Methylaethylacroleïn erhalten ist und die Entstehung leicht erklärbar ist, wenn man annimmt, dass $2\left(\mathrm{C}_{6} \mathrm{H}_{10} \mathrm{O}\right)+\mathrm{NH}_{3}=\mathrm{C}_{12} \mathrm{H}_{19} \mathrm{~N}+2 \mathrm{H}_{2} \mathrm{O}$ geben, während wohl schwer eine Entstehungsgleichung für das Product $\mathrm{C}_{11} \mathrm{H}_{17} \mathrm{~N}$ sich finden lassen dürfte.

Die höher siedenden Fractionen des auf $200^{\circ}$ erhitzten Productes sind dunkelgelbe bis schwarze, zähe, dicke Flüssigkeiten, aus denen ich kein weiteres Product mehr abscheiden konnte.

\section{Fraction 165 bis $175^{\circ} \mathrm{C}$.}

Hingegen gelang es mir aus der ersten Fraction 165 bis $175^{\circ} \mathrm{C}$, das ist einer Vorfraction des Parvolin, einen Körper in seinem Platindoppelsalze zu isoliren.

Ich löste das Basengemisch, welches diese Fraction bildete, in Salzsäure und fällte fractionirt mit Platinchlorid; von vier Fractionen, die ich erhielt, besass die erste den Schmelzpunkt $254^{\circ}$, die zweite $252^{\circ}$, die dritte $230^{\circ}$, die letzte aus den Mutterlaugen gewonnen $188^{\circ}$.

Die Analysen der ersten Fraction zeigten, dass ein kohlenstoffärmerer Körper als Parvolin vorliege, während die vierte Fraction den Schmelzpunkt $188^{\circ}$ aufwies, also muthmasslich Parvolinplatindoppelsalz war.

Da bei der Fällung anfangs andere Krystalle fielen, als später bei längerem Stehen sich in der Mutterlauge zeigten, so basirte ich hierauf und auf die grosse Verschiedenheit der Schmelzpunkte die Trennung der Platinsalze, und es gelang in der That nach oftmaligem Umkrystallisiren aus heissem Wasser zu einem schön krystallisirten, tiefgelben Platindoppelsalze zu kommen, das den Schmelzpunkt von $258^{\circ}$ beibehielt.

Die Analysen ergaben folgende Zahlen:

I. $0 \cdot 2100 g$ Substanz lieferten bei der Verbrennung $0 \cdot 1846 g$ Kohlensäure, $0 \cdot 0576 g$ Wasser und hinterliessen am Platinmantel, wit dem das Schiffchen umgeben war, aussergewöhnlich stark zerstäubt, $0 \cdot 0674 g$ Platin.

II. $0 \cdot 1279 g$ Substanz lieferten beim Glühen im geschlossenen Tiegel $0 \cdot 0415 \mathrm{~g}$ Platin. 
In 100 Theilen:

\begin{tabular}{|c|c|c|}
\hline \multicolumn{2}{|c|}{ Gefunden } & \multirow{2}{*}{$\begin{array}{c}\begin{array}{c}\text { Berechnet fuir } \\
\left(\mathrm{C}_{6} \mathrm{H}_{7} \mathrm{~N} . \mathrm{HCl}\right)_{2} \mathrm{PtCl}_{4}\end{array} \\
\end{array}$} \\
\hline I. & II. & \\
\hline $.32 \cdot 09$ & $32 \cdot 45$ & $32 \cdot 66$ \\
\hline $.23 \cdot 98$ & - & $24 \cdot 18$ \\
\hline asserstoff . . 3.04 & 一 & $2 \cdot 69$ \\
\hline
\end{tabular}

Eine Platinbestimmung der vierten Fällungsfraction bestätigte die Vermuthung, dass Parvolinplatinsalz anfangs beigemengt gewesen war.

$0 \cdot 2945 \mathrm{~g}$ Substanz gaben beim Glühen $0.0851 \mathrm{~g}$ Platin oder $28 \cdot 89 \%$ Platin gegen $28 \cdot 57 \%$, welche sich für Parvolinplatinsalz $\left(\mathrm{C}_{9} \mathrm{H}_{13} \mathrm{~N} . \mathrm{HCl}\right)_{2} \mathrm{PtCl}_{4}$ berechnen.

Die Übereinstimmung der gefundenen und der für dasPicolinplatinsalz berechneten Procentzahlen ist eine gute, und der erhöhte Siedepunkt der freien Base $165-175^{\circ}$ erklärlich, da wie die Mengen der gewonnenen Platinsalze zeigten - ein Gemisch von beiläufig einem Fünftel des kohlenstoffärmeren Körpers (Picolin) mit vier Fünfteln des kohlenstoffreicheren Parvolin vorhanden war; endlich stehen die Krystallmessungen, welche Herr Dr. Bř ezin a die Güte hatte auszuführen, in genähertem Einklange mit Messungen von Picolinplatinsalzen, welche er fürr Weidel gemessen hatte, so dass man wohl annehmen darf, dass wirklich bei der Einwirkung von Ammoniak auf Methylaethylacroleïn neben Parrolin und der Base $\mathrm{C}_{12} \mathrm{H}_{19} \mathrm{~N}$ auch ein

$$
\text { Picolin: } \mathrm{C}_{6} \mathrm{H}_{7} \mathrm{~N}
$$

entstanden sei. 